\title{
"Privat vor Staat"? Zur Entwicklung politischer Leitbilder über die Rolle des Staates
}

Hans-Jürgen Bieling

\begin{abstract}
Nachdem lange die These vom Rückzug des Staates en vogue war, wird seit einiger Zeit wieder intensiv über die Rückkehr des Staates diskutiert. Ob es um Mindestlöhne, um die Bildung und Kindererziehung, um die Sicherung eines funktionsfähigen Kreditsystems oder um die Bewältigung der Weltwirtschaftskrise geht, eine starke Rolle des Staates wird in der öffentlichen Diskussion inzwischen wieder recht positiv, jedenfalls als erforderlich gesehen. Gleichzeitig gibt es jedoch Zweifel daran, ob der allgemeine „Stimmungsumschwung“ bereits auf einen erneuten „Paradigmenwechsel“ staatlicher Politik verweist. Diese Ungewissheit in Rechnung gestellt, wird nachfolgend der Versuch unternommen, aus dem historischen Wandel staatlicher Leitbilder einige Erkenntnisse für deren praktische Relevanz und zukünftige Entwicklung zu gewinnen.
\end{abstract}

\section{Leitbilder in der Politik}

Leitbildern kommt in der Politik eine ganz zentrale Bedeutung zu. Nach Heinrich Schneider (1992) repräsentieren sie ein dialektisch aufeinander bezogenes Ensemble von Zielvorstellungen sowie Wahrnehmungs- und Deutungsmustern, die konkreten Situationen eine bestimmte Relevanzstruktur zuschreiben. Von einem Leitbildwandel kann dann gesprochen werden, wenn vor dem Hintergrund eines veränderten gesellschaftlichen Problemhorizonts politische Zielsetzungen grundlegend neu definiert werden. Die Transformation von politischen Leitbildern oder Paradigmen erschließt sich demzufolge durch das spezifische Zusammenspiel von allgemeinen materiellen Veränderungen und deren diskursiv-ideeller Bearbeitung. Sie ist in dem Maße umkämpft, wie - insbesondere in Krisenzeiten - zumeist mehrere Leitbilder miteinander konkurrieren. Welches Leitbild sich durchsetzt, ist oft nur schwer vorherzusehen. Ihre politische Gestaltungskraft wächst grundsätzlich in dem Maße, wie die unterschiedlichen Ebenen der Leitbildproduktion kohärent ineinandergreifen und sich wechselseitig verstärken. Vereinfacht lassen sich drei Ebenen oder "Stockwerke“ der Leitbild(re-)produktion unterscheiden (Hall 1989, S. 80ff.):

(1) Das oberste Stockwerk bilden die wissenschaftlich begründeten Entwürfe von konzeptionell arbeitenden Intellektuellen oder Think Tanks. Sie zeichnen sich durch eine relativ schlüssige, argumentativ kohärente Perspektive aus, mit der die jeweiligen Themen oder Problemgegenstände analysiert und interpretiert werden. Der wissenschaftliche Objektivitätsanspruch und die öffentliche Reputation der Urheber oder Initiatoren spezifischer Diskurse spielen nicht selten eine wichtige Rolle.

(2) Unterhalb der Ebene der theoretischkonzeptionellen Überlegungen sorgen sogenannte "second hand dealers in ideas" (Hayek zit. nach Cockett 1994, S. 159) dafür, dass die großen intellektuellen Entwürfe in der öffentlichen Diskussion aufgegriffen, bildungspolitisch verbreitet und in der politischen Praxis operationalisiert, d. h. in konkrete Projekte und Initiativen übersetzt werden. Journalisten, Lehrer, Partei- und Verbandspolitiker und andere meinungsformende Berufsgruppen wirken darauf hin, dass Ideen und Leitbilder aufgenommen und praktisch wirksam werden. Die systematischen und elaborierten Überlegungen der konzeptionell arbeitenden Intellektuellen werden dabei zum Teil stark vereinfacht. Oft müssen zudem Konzessionen gemacht werden, um historisch tradierte institutionelle, kulturelle und machtpolitische Widerstände abzuschwächen. Mit anderen Worten: Das zweite oder mittlere Stockwerk ist durch beträchtliche Kompromisszwänge und eine geringere Kohärenz der leitbildgeprägten Diskurse gekennzeichnet.
(3) Noch schwächer ist diese Kohärenz auf dem untersten Stockwerk, dem Alltagsbewusstsein der breiten Massen. Dies liegt grundsätzlich daran, dass das Alltagsbewusstsein zwar nach wie vor stark durch die soziale (Klassen-)Lage bestimmt ist, zugleich aber - verstärkt durch die Erosion sozialmoralischer Milieus und die massenmediale Kommunikation - in den alltäglichen Diskursen häufig sehr disparate oder sogar gegensätzliche Ereignisse, Theoreme und Erfahrungen nebeneinander koexistieren. Angesichts der begrenzten Möglichkeiten einer stetigen, theoretisch angeleiteten Reflexion ist das Alltagsbewusstsein meist stark fragmentiert und nicht selten für Teilelemente ganz unterschiedlicher Leitbilder empfänglich.

Welche Leitbilder gesellschaftspolitisch relevant oder sogar hegemonial werden, $d$. h. eine breite Zustimmung und aktive Unterstützung finden, ist demzufolge nicht nur von der Brillanz des intellektuellen Designs und dem Engagement der meinungsbildenden Berufsgruppen abhängig. Gerade das letztgenannte Stockwerk, das Alltags-

\footnotetext{
Hans-Jürgen Bieling, $P D$, Dr., Vertretungsprofessor am Fachbereich Sozialökonomie der Universität Hamburg. Arbeitsschwerpunkte: Europäische Integration, Internationale Politische Ökonomie, Politik-, Staatsund Gesellschaftstheorie. e-mail: Hans-Juergen.Bieling@wiso.unihamburg.de
} 
bewusstsein, zeigt sehr deutlich, dass auch die Prozesse der sozioökonomischen Restrukturierung und politisch-institutionellen Reorganisation, vor allem die mit ihnen verbundenen Erfahrungen - Vorteile wie Enttäuschungen - und Erwartungen, die gesellschaftliche Resonanz spezifischer Leitbilder beeinflussen.

Daher ist es auch wenig verwunderlich, dass sich Leitbilder, insbesondere die des Staates, im Kontext gesellschaftlicher und politisch-institutioneller Transformationsund Krisenprozesse immer wieder verändern. Von zentraler Bedeutung ist dabei das Zusammenspiel von Krise, Kritik und intellektueller Intervention (Kosellek 1973; Steil 1993). Krisen sind definitionsgemäß Phasen tiefer Unsicherheit, also Zeiträume der Entscheidung oder Wende, in denen medizinisch betrachtet - im Krankheitsverlauf der Patient entweder gesundet oder stirbt oder allgemeiner formuliert: „das Alte stirbt (während) ... das Neue ... noch nicht zur Welt kommen (kann)" (Gramsci 1991ff., S. 354). Für Intellektuelle unterschiedlichen Typs offerieren Krisen nicht nur die Chance, dass ihre Kritik an den gegebenen Verhältnissen und (hegemonialen) Leitbildern auf öffentliche Zustimmung stößt. Krisen bieten auch die Gelegenheit, die alten Leitbilder - Überzeugungen und gesellschaftliche Praxen - abzulösen und durch neue zu ersetzen. Die intellektuellen Krisendiagnosen und die Kritik der bestehenden Ordnungsmuster und Paradigmen leiten oft recht unmittelbar über zu alternativen Leitbildern. $\mathrm{Ob}$ diese weithin Unterstützung finden, ist keineswegs gewiss. Grundsätzlich gilt jedoch, dass sich die Erfolgsaussichten in dem Maße verbessern, wie die neuen Leitbilder - einschließlich ihrer politischen Implikationen - als notwendige, angemessene, wenn möglich sogar allseits vorteilhafte Antwort auf akute Krisen und drängende gesellschaftliche Probleme präsentiert werden können.

\section{2 \\ Leitbilder und Projekte einer transformierten Staatlichkeit}

Die Attraktivität, die Leitbilder in der öffentlichen Wahrnehmung genießen, ist nicht einfach gegeben, sondern fortwährend politisch und diskursiv umkämpft.
Was dies konkret bedeutet, soll nachfolgend anhand der Abfolge einiger konkurrierender Leitbilder - vom keynesianischen Wohlfahrtsstaat über den schlanken Wettbewerbsstaat bis zum aktivierenden Staat und Gewährleistungsstaat - knapp rekonstruiert werden.

\subsection{VOM KEYNESIANISCHEN WOHLFAHRTSSTAAT ZUM SCHLANKEN WETTBEWERBSSTAAT}

Das Leitbild des keynesianischen Wohlfahrtsstaates war gleichsam strukturell in die Nachkriegsordnung des sogenannten eingebetteten Liberalismus (Ruggie 1982) eingeschrieben. Nicht nur die Architekten des Bretton-Woods-Systems, auch einflussreiche gesellschaftliche Kräfte-(Volks-)Parteien, Gewerkschaften, Arbeitgeberverbände, Kirchen etc. - vertraten nach dem Zweiten Weltkrieg die Auffassung, dass eine liberale Weltwirtschaftsordnung - wenn sie denn mit demokratischen gesellschaftlichen Organisationsformen in Einklang gebracht werden sollte - mehrfach einzuhegen sei: zum einen durch eine politische Kontrolle und Gestaltung der internationalen Währungs- und Finanzbeziehungen und zum anderen durch wohlfahrtsstaatliche und korporatistische Institutionen des sozialen Interessenausgleichs. Die Lohnsteigerungen und wohlfahrtsstaatlichen Leistungen waren zugleich insofern sehr bedeutsam, als durch sie das fordistische Produktionsmodell - Massenproduktion und Taylorismus - sowie die Prozesse der „inneren Landnahme“ (Lutz 1984) nachfrageseitig abgestützt wurden.

Ungeachtet dieser Funktionalität wurde das Leitbild des keynesianischen Wohlfahrtsstaates in Deutschland erst relativ spät, gegen Ende der 1960er Jahre, prominent. Es trat in dem Augenblick in den Vordergrund, als ökonomische und soziale Krisenprozesse durch die von Karl Schiller entwickelte Globalsteuerungskonzeption sowie durch die Ausweitung wohlfahrtsstaatlicher Leistungen, die Bildungsexpansion oder die Reform der betrieblichen Mitbestimmung aktiv bearbeitet wurden. Als Kontrapunkt zur aufkommenden staatlichen Planungseuphorie geriet der keynesianische Wohlfahrtsstaat jedoch bereits früh in die Kritik. Auf der einen Seite wandten zunächst linke Kritiker ein (Offe 1972; Habermas 1973), dass der keynesianische Wohlfahrtsstaat zwar die sozioökonomischen Krisen spätkapitalistischer Vergesell- schaftung bewältigen kann, sich hierdurch aber die Krisenprozesse ins politische System verlagern; und zwar in dem Maße, wie die wohlfahrtsstaatliche Regulierung und Krisenintervention besonders legitimationsbedürftig ist, sich ihr bürokratischer Charakter aber nur unzureichend mit soziokulturellen lebensweltlichen Idealen und partizipativen Kontroll- und Mitgestaltungsansprüchen in Übereinstimmung bringen lässt. Auf der anderen Seite sahen wenig später die rechten Vertreter der Unregierbarkeitsthese das Problem keineswegs in der unzureichenden demokratischen Kontrolle des Wohlfahrtsstaates, sondern vielmehr in dessen expansiver Dynamik (Hennis 1976; Matz 1978). Im Zusammenspiel von gesellschaftlicher Anspruchsinflation und staatlichem Machbarkeitswahn würde der Staat letztlich überfordert und tendenziell unregierbar; zudem würden auch die Marktkräfte unterdrückt.

Bereits im Laufe der 1970er Jahre, spätestens jedoch im Übergang zu den 1980er Jahren gewann die rechte Kritik und die ihr zugrundeliegende Krisendiagnose in der öffentlichen politischen Diskussion an Einfluss. Dies lag nicht zuletzt daran, dass sich - in den OECD-Staaten insgesamt, so auch in der Bundesrepublik Deutschland - die politökonomischen Entwicklungsperspektiven verändert hatten. Der keynesianische Wohlfahrtsstaat und zu starke Gewerkschaften galten weithin als Verursacher der Stagflation - d. h. einer wirtschaftlichen Stagnation in Kombination mit relativ hohen Inflationsraten - und der zurückgekehrten Massenerwerbslosigkeit. Zudem war die Deutsche Bundesbank Mitte der 1970er Jahre auf eine relativ strikte AntiInflationspolitik eingeschwenkt, was eine keynesianische Nachfragestimulierung erschwerte, wenn nicht sogar unmöglich machte. Der diskursive und politische Erfolg marktliberal-angebotsorientierter Modernisierungskonzepte hatte mithin im Scheitern zuvor praktizierter keynesianischer Strategien seine Grundlage und schien mit der scharfen Kritik am bürokratisch organisierten, jedenfalls zu generösen Wohlfahrtsstaat und der durch die Gewerkschaften geförderten Überregulierung der Arbeitsmärkte auch eine passende, zukunftsgerichtete Antwort zu präsentieren.

Die Kritik am keynesianischen Wohlfahrtsstaat stützte sich nun freilich nicht allein auf die Überlegungen neoliberaler Ökonomen, die sich bereits seit Ende der 1940er 
Abb. 1: Entwicklung der Staatsquote

- Staatsausgaben in \% des Bruttoinlandsprodukts -

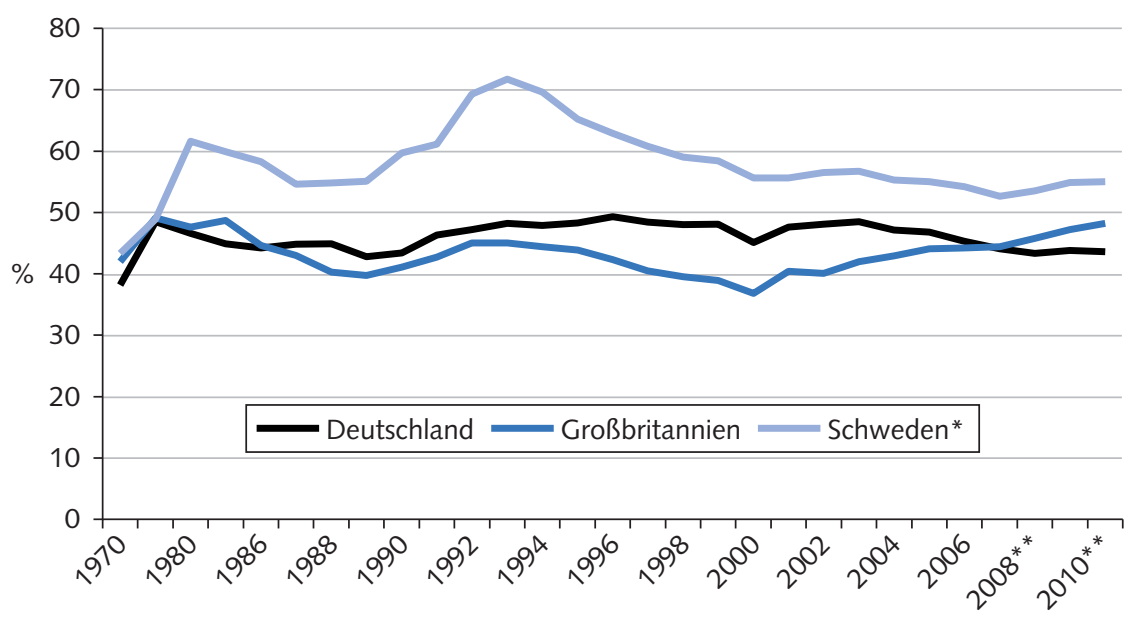

Quelle: Europäische Kommission (2008, S. 178f.); *für Schweden: bis 1992; * * geschätzt OECD Economic Outlook Nr. 40 (1986), Nr. 68 (2000).

\section{WSI MITTELUNGeN}

Jahre in diversen Netzwerken zusammengeschlossen und politisch positioniert hatten (Cockett 1994; Plehwe/Walpen 1999). Auch Intellektuelle aus den Gebieten der Soziologie und Philosophie trugen aktiv mit dazu bei, die gesellschaftliche Rezeption von neokonservativ-neoliberalen Ideen zu fördern (Bell 1991). So unterschiedlich die Begründungen im Einzelnen auch waren, so klar war doch ihr gemeinsamer Fokus: An die Stelle des bevormundendbürokratischen, kostenintensiven und leistungsfeindlichen Wohlfahrtsstaates müsse ein effizienter und wettbewerbsorientierter, kurzum schlanker Wettbewerbsstaat treten, der sich auf seine unmittelbaren Kernaufgaben konzentriert und zugleich der individuellen Selbstverantwortung, vor allem den Selbstheilungskräften des Marktes und der Privatinitiative des freien Unternehmertums, wieder neue Handlungsräume eröffnet.

\subsection{VOM SCHLANKEN WETTBEWERBSSTAAT ZUM AKTIVIERENDEN STAAT}

Gestützt auf die Projekte der Verwaltungsmodernisierung, also des New Public Management, wurde das Leitbild des schlanken Staates freilich erst im Laufe der 1990er Jahre in Anlehnung an die zwischenzeitlich sehr populären Konzepte der lean production und des lean management formuliert. Obwohl dieses Leitbild in der deutschen Diskussion zunächst keine Rolle spielte und auch später keineswegs hegemonial wurde, markierten die ihm eingeschriebenen Perspektiven der finanzpolitischen Konsolidierung, der arbeits- und sozialpolitischen Flexibilisierung und Deregulierung sowie der Entstaatlichung zentrale Referenzpunkte der seit den 1980er Jahren praktizierten Reorganisationsstrategie. Diese Reorganisationsstrategie war zweifelsohne Ausdruck eines Paradigmenwechsels. Die Staatsorganisation stand nicht mehr im Zeichen einer - soziale Ungleichheiten, Unsicherheiten und Ausgrenzungseffekte aktiv bekämpfenden - Politik der sozialen Anrechte, sondern unterlag zunehmend einer marktorientierten Leistungs- und Effizienzkonzeption, der zufolge mit Blick auf das übergeordnete Ziel einer verbesserten internationalen Wettbewerbsfähigkeit andere staatliche Aufgabenbereiche einer Kompatibilitätsprüfung unterzogen wurden. Dies ist nicht so zu verstehen, dass nicht auch andere, unter anderem repressive Formen der Staatstätigkeitz. B. die informationstechnologische Überwachung, die restriktive Migrationskontrolle oder die Bekämpfung von Kriminalität - nach wie vor bedeutsam waren oder sogar aufgewertet wurden (Kannankulam 2008, S. 308ff.). Die politischen Diskurse orientierten sich jedoch sehr stark am übergeordneten Ideal eines schlanken Wettbewerbsstaates, der sich primär auf seine Kernkompetenzen - die Garantie von Eigentumsrechten, die Ermöglichung und Förderung des Wettbewerbs sowie die Organisation der inneren und äußeren Sicherheit - konzentriert und unnötigen wirtschafts- und sozialpolitischen „Ballast“ abwirft (Jessop 1993; Hirsch 1998, S. 28ff.).

In der politischen Praxis stieß das Leitbild des schlanken Wettbewerbsstaates jedoch nicht nur auf Zustimmung, sondern auch auf vielfältige Widerstände. $\mathrm{Zu}$ erwähnen sind unter anderem die Beharrungskraft der tradierten wohlfahrtsstaatlichen Institutionen, die korporatistischen Netzwerke und gewerkschaftlichen Proteste, die bremsende Wirkung des föderalen Systems und auch die - infolge der Deregulierung, der Beschäftigungskrise und der deutschen Einheit - gestiegenen sozialen Lasten. So stellt sich das Gesamtbild der 1980er und 1990er Jahre zumindest ambivalent dar. Einerseits ist, was die Inflationsbekämpfung, die Senkung der Unternehmenssteuern, die Reform der Arbeitsmärkte und Sozialsysteme und auch die Prozesse der Privatisierung und Entstaatlichung betrifft, durchaus eine wettbewerbsorientierte Veränderungsdynamik erkennbar (Dräger 2004). In vielen Ländern, so auch in Deutschland, sind z. B. die Kriterien für die Inanspruchnahme sozialer Leistungen restriktiver definiert und die Arbeitsmärkte partiell dereguliert worden. Andererseits klafft zwischen dem bekundeten Verschlankungsanspruch und der praktischen Politik jedoch eine unübersehbare Lücke. So gelang es der Regierung Kohl zwar, ab Anfang der 1990er Jahre den seit Langem proklamierten Entstaatlichungs- bzw. Privatisierungsprozess voranzutreiben, ohne hierdurch allerdings den erneuten - durch die deutsche Einheit und das schwache Wirtschaftswachstum begünstigten - Anstieg der Staatsquote verhindern zu können ( $A b$ bildung 1).

Seit Mitte der 1990er Jahre beschleunigte sich dann freilich der Entstaatlichungsprozess (Bofinger 2008; Bieling 2008: 542ff; Engartner 2008), obwohl das Leitbild des schlanken Wettbewerbstaates allmählich wieder in den Hintergrund trat. Die Ursachen für diese Entwicklung waren vielschichtig. Drei Faktoren kam jedoch eine besondere Bedeutung zu:

(1) Im Laufe der 1990er Jahre machten sich die Folgen der intensivierten europäischen Integration bemerkbar (Bieling et al. 2008; Brandt et al. 2008). So wurden im Zuge der Bestrebungen, den EG-Binnenmarkt zu vertiefen, viele Infrastrukturdienstleistungen - z. B. in den Bereichen Post, Telekommunikation, Bahn oder auch der Stromund Gasversorgung - liberalisiert und zum 
Teil auch privatisiert. Die Europäische Union ging zwar nicht so weit, eine Privatisierung vorzuschreiben, legte diese jedoch insofern nahe, als im Binnenmarkt staatlich organisierte Wettbewerbsverzerrungen unterbunden und die nationalen Haushalte mit Blick auf die Wirtschafts- und Währungsunion (WWU) konsolidiert werden sollten.

(2) Seit der zweiten Hälfte der 1990er Jahre sorgte auch die Finanzmarktdynamik, die durch den Integrationsprozess ebenfalls gefördert wurde, dafür, dass sich die Bereitschaft der nationalen Regierungen, so auch der deutschen, erhöhte, bisherige Staatsunternehmen zu privatisieren. Steigende Aktienpreise und Privatisierungserlöse standen in einem positiven Wechselverhältnis. Wie die Erwartung steigender Kurswerte die Privatisierungsaktivitäten der Regierung - insbesondere des Finanzministers - stimulierte, so trug die Privatisierung ihrerseits mit dazu bei, zusätzliches Kapital zu mobilisieren und die Aktienkurse nach oben zu treiben.

(3) Schließlich entfaltete sich parallel zu den ersten beiden Faktoren ein neuer Krisendiskurs, der ungeachtet aller Liberalisierungs-, Deregulierungs- und Privatisierungsschritte einen anhaltenden Reformstau identifizierte. Dieser Reformstau, so das vielfach vorgebrachte Argument, resultiere vor allem daraus, dass die Revitalisierung von Ökonomie und Gesellschaft weniger einen schlanken, sich weitgehend zurückziehenden, als vielmehr einen aktivierenden Staat benötige.

Das neue Leitbild des aktivierenden Staates war nicht zuletzt durch die Schriften des britischen Intellektuellen Anthony Giddens (1994, S. 174ff.) prominent geworden. Aber auch einige deutsche Wissenschaftler (Bandemer et al. 1995; Blanke/Bandemer 1999) im Umfeld der Sozialdemokratie des Dritten Wegs hatten sich sehr positiv auf das Leitbild des aktivierenden Staates bezogen. Im Vorfeld der Bundestagswahl 1998 verwiesen die Befürworter des aktivierenden Staates wiederholt auf die wirtschafts-, sozial- und arbeitsmarktpolitischen Reformprozesse in Dänemark, Großbritannien und den Niederlanden, mehr noch: stilisierten diese zuweilen sogar zu Modellen, an denen sich die deutsche Politik zu orientieren habe. Jenseits kleinerer Differenzen waren sich die Protagonisten des akti- vierenden Staates darin einig, dass dieser gemäß dem Slogan eines neuen Dritten Wegs jenseits der alten Leitbilder des keynesianischen Wohlfahrtsstaates auf der einen und des Minimalstaats bzw. schlanken Wettbewerbsstaats auf der anderen Seite darauf ausgerichtet sein sollte, durch eine kooperative Verhaltenssteuerung der Staatsbürger und Marktteilnehmer zusätzliche gesellschaftliche Produktivitätspotenziale zu mobilisieren:

- unter anderem durch den Aufbau und die Stärkung kooperativer Dialog- und Verhandlungsarenen von staatlichen und zivilgesellschaftlichen Akteuren;

- durch die Neudefinition der von staatlichen und privaten Akteuren zu erbringenden gesellschaftlichen Leistungen;

- durch ein neues Arrangement von politischen Verantwortlichkeiten, d. h. durch die Aufwertung von bürgerschaftlichem Engagement und die staatliche Förderung von Eigenverantwortlichkeit.

\subsection{VOM LEISTUNGS- ZUM GEWÄHRLEISTUNGSSTAAT}

Seit Ende der 1990er Jahre kristallisierte sich mit dem Gewährleistungsstaat schließlich ein weiteres Leitbild heraus, das die Funktionsweise des aktivierenden Staates in wichtigen Punkten - mit Blick auf die Entstaatlichung und Verwaltungsmodernisierung - akzentuiert und präzisiert. Mit dem Leitbild des Gewährleistungsstaates wird unterstellt, dass der alte „Produktionsoder Leistungsstaat", der noch selbst die Bereitstellung der öffentlichen Infrastruktur organisiert hat, den Anforderungen einer modernisierten, den privaten Unternehmen und dynamischen Marktkräften neuen Raum gebenden Wirtschaftsordnung nicht mehr entspricht. Um die ökonomischen Produktivitätspotenziale abzurufen und nutzbar zu machen, so die weitere Argumentation, sei es sehr viel besser, wenn das Angebot öffentlicher Versorgungsleistungen in weiten Bereichen zukünftig von privatwirtschaftlichen Betrieben erbracht wird, wobei dem Staat allerdings nach wie vor die Aufgabe zufällt, eine flächendeckende Versorgung, eine ausreichende Qualität und ein angemessenes Preisniveau der jeweiligen Dienste zu garantieren. Dem Leitbild des Gewährleistungsstaates liegt mithin die Idee zugrunde, dass sich mit Blick auf die Reorganisation öffentlicher Dienstleistungen der Staat vom Versorgungsmonopolisten zum Versorgungsmanager wandelt. Gemäß der neuen Arbeits- und Verantwortungsteilung mit der Privatwirtschaft soll sich der Staat fortan auf die rechtlich-regulative Einfassung und politisch-administrative Kontrolle des Dienstleistungsangebots beschränken (Schuppert 2008).

Auf den ersten Blick scheint es sich bei der Debatte über den Gewährleistungsstaat - allein schon aus begrifflichen Gründen um eine spezifisch deutsche Diskussion zu handeln, die vor allem von Wissenschaftlern wie Christoph Reichard, Gunnar Folke Schuppert oder Edgar Grande geführt wurde. Genauer betrachtet ist diese Charakterisierung aber nicht ganz zutreffend. Schließlich ist im Kontext der Liberalisierung und Privatisierung vormals öffentlicher Dienstleistungsunternehmen auch in anderen Ländern oder der Europäischen Union (EU) über den Neuzuschnitt staatlicher Aufgaben und Kompetenzen intensiv diskutiert und geforscht worden. Im Unterschied zur normativ $-d$. h. verantwortungsethisch - aufgeladenen Konzeption des Gewährleistungsstaates wurde dabei zumeist sehr viel nüchterner und funktionalistisch vom Regulierungsstaat (regulatory state) gesprochen (Majone 1996). Dessen Aufgaben umfassen zum einen die Prozesse der Marktöffnung und Wettbewerbsregulierung, zum anderen aber auch die inhaltliche Qualitätsregulierung, durch die unter Befolgung gewisser Kriterien eine breite und niveauvolle Mindestversorgung gesichert werden soll.

Das Leitbild des Regulierungs- oder Gewährleistungsstaates ist eng verknüpft mit einer zwar wettbewerbs- und sozialpolitisch eingefassten, aber fortgesetzten Entstaatlichungspolitik. Dies heißt, die bereits durch das Leitbild des schlanken Wettbewerbsstaates propagierte Auffassung, dass eine private der staatlichen Leistungserbringung grundsätzlich überlegen sei, wurde durch das Leitbild des Gewährleistungsstaates allenfalls punktuell der öffentlichen Diskussion ausgesetzt. Zugleich verschob sich mit dem Erfolg, d. h. der Ausstrahlungskraft des Leitbildes, tendenziell das Terrain der politischen Auseinandersetzung. Ging es vormals noch um die grundsätzliche Frage, ob Privatisierung der richtige Weg ist, so richtete sich im Rahmen der Gewährleistungskonzeption der Blick zunehmend nur noch darauf, wie Privatisierung und Regulierung gestaltet werden sollten, also auf die Konditionen und die 


\begin{tabular}{|c|c|c|c|c|c|c|}
\hline Branchen & Insgesamt & CDU/CSU & SPD & FDP & Grünen & Linke \\
\hline Energiewirtschaft & 77 & 73 & 83 & 70 & 84 & 78 \\
\hline Banken/Versicherungen & 64 & 61 & 76 & 58 & 67 & 73 \\
\hline Fluglinien, Bahn, Post & 60 & 58 & 70 & 46 & 74 & 66 \\
\hline Chemie/Pharma & 45 & 39 & 51 & 35 & 54 & 53 \\
\hline Telekommunikation & 40 & 39 & 41 & 38 & 38 & 46 \\
\hline Landwirtschaft & 40 & 33 & 37 & 25 & 44 & 42 \\
\hline Elektroindustrie & 35 & 35 & 34 & 26 & 33 & 33 \\
\hline Immobilienwirtschaft & 31 & 23 & 40 & 28 & 37 & 26 \\
\hline Automobilindustrie & 26 & 24 & 26 & 23 & 29 & 23 \\
\hline
\end{tabular}

operative Umsetzung der wettbewerbsund sozialpolitischen Kontrollregeln. Darüber hinaus wurden die strukturellen Faktoren, die den Entstaatlichungsprozess begünstigt haben - die Finanzkrise der öffentlichen Haushalte, der vielfach beobachtbare Verfall der öffentlichen Infrastruktur, europäische Liberalisierungsdirektiven und dynamische Aktienmärkte -, politisch kaum infrage gestellt.

\subsection{AUF DEM WEG ZU EINEM NEUEN INTERVENTIONSSTAAT?}

Die staatliche Selbstbescheidung und durchgreifende Akzeptanz der entfesselten Wettbewerbs- und Privatisierungsdynamik scheint inzwischen nicht mehr die politische Agenda zu bestimmen. In gewisser Weise vollzog sich bereits im Bundestagswahlkampf des Jahres 2005 eine Kehrtwende. Entgegen der zuvor im Rahmen der Agenda 2010 praktizierten Politik wurden die Vertreter der rot-grünen Koalition nicht müde, sich als Repräsentanten einer solidarischen Reformpolitik zu präsentieren; und die Berufung von Paul Kirchoff in das Schattenkabinett von Angela Merkel hatte der CDU/CSU deutlich vor Augen geführt, dass sich mit einer flat-tax als Ausdruck einer marktliberalen Reformstrategie keine konservativ-liberale Parlamentsmehrheit organisieren lässt.

Diese Ereignisse verweisen zugleich auf einen Wandel des gesellschaftlichen Krisendiskurses. Nicht mehr der Reformstau, sondern die sich mehrenden sozialen Probleme - also Phänomene wie Armut trotz Arbeit und die voranschreitende Prekarisierung - bestimmten zunehmend die politische Szenerie. Um mit Karl Polanyi (1977) zu sprechen: Der marktliberale Reformeifer, der sich am Prinzip eines sich weitgehend selbst regulierenden Marktes orientierte, hatte inzwischen viele negative soziale Folgewirkungen produziert, die esgemäß dem Prinzip der sozialen Protektion der Gesellschaft - nun politisch zu korrigieren galt. Getragen vom globalen Wirtschaftsaufschwung schlug in der deutschen Regierungspolitik entsprechend das Pendel zurück. Dies betraf weniger die Steuer- und Finanzpolitik, wo die Konsolidierung des Staatshaushaltes nach wie vor Priorität hatte und durch die Erhöhung der Mehrwertsteuer unterstützt werden sollte, als vor allem die Arbeitsmarkt- und Sozialpolitik. Um nur einige Beispiele zu nennen:

(1) Die Hartz-IV-Gesetzgebung wurde insofern korrigiert, als für ältere Arbeitnehmer der Bezug von Arbeitslosengeld verlängert wurde;

(2) auf den expandierenden Niedriglohnsektor wurde - zumindest partiell - mit der regierungspolitisch geförderten Aushandlung von Mindestlöhnen reagiert;

(3) die Reformvorschläge der bundesdeutschen Familienministerin Ursula von der Leyen zur Erhöhung des Kindergeldes, zu Elterngeld und Elternteilzeit sowie zur Verbesserung der öffentlichen Kinderbetreuung weisen in die Richtung einer gestärkten sozialstaatlichen Flankierung der marktliberalen Wirtschafts- und Arbeitsmarktpolitik.

Das Leitbild des Gewährleistungsstaates blieb von diesen Veränderungen in der Arbeits- und Sozialpolitik zunächst unberührt. Dies lag vermutlich daran, dass die Volks- oder Bürgerbegehren, durch die auf der Landes- und Kommunalebene die Privatisierung von Infrastruktur- und Versorgungsbetrieben in den vergangenen Jahren politisiert wurde (Mittendorf 2008), in der öffentlichen Diskussion oft nur eine marginale Rolle spielten. In den letzten Mona- ten hat sich dies allerdings deutlich verändert. Zum einen erzeugten zwei Forsa-Umfragen - eine Ende 2007, die andere im Sommer 2008 (taz, 9. Januar 2008; Handelsblatt, 8. Oktober 2008) -, die eine privatisierungsablehnende Grundhaltung der deutschen Bevölkerung zum Ausdruck brachten, für ein recht breites Medienecho; und zum anderen haben die sehr kontrovers geführten Diskussionen über den Börsengang der Deutschen Bahn AG die allgemeine Sensibilisierung für die - oft negativen - Folgen eines privatisierten öffentlichen Dienstleistungsangebots gefördert. Mit anderen Worten: Es hat sich mittlerweile die Auffassung breitgemacht, dass der Gewährleistungsstaat vielfach nicht mehr in der Lage sei, flächendeckende, zuverlässige, qualitativ hochwertige und preisgünstige Infrastrukturdienstleistungen sicherzustellen.

Die Verschiebung, vielleicht sogar Aufhebung des Börsengangs der Deutschen Bahn AG war jedoch weniger der Privatisierungsskepsis der Bevölkerung - und der Beschäftigten in den öffentlichen Versorgungsunternehmen - geschuldet, als vielmehr der sich zuspitzenden Finanzmarktkrise. Angesichts fallender Aktienkurse, also der Erwartung geringer Privatisierungserlöse, ließ sich der Börsengang politisch nicht mehr vermitteln und legitimieren. Generell scheint sich die Finanzmarktkrise als erneuter Wendepunkt in der Definition staatlicher Aufgabenbereiche zu erweisen. Jürgen Habermas (2008) formulierte: „Blamiert hat sich die Agenda, die Anlegerinteressen eine rücksichtslose Dominanz einräumt, die ungerührt wachsende soziale Ungleichheit, das Entstehen eines Prekariats, Kinderarmut, Niedriglöhne und so weiter in Kauf nimmt, die mit ihrem Privatisierungswahn Kernfunktionen des Staates aushöhlt, die die deliberativen Reste der politischen Öffentlichkeit an renditesteigernde Finanzinvestoren verscherbelt, Kultur und Bildung von den Interessen und Launen konjunkturempfindlicher Sponsoren abhängig macht."

Doch nicht nur die Perspektive eines renommierten Intellektuellen, auch die öffentliche Stimmungslage hat sich im Kontext der Finanzkrise partiell radikalisiert. Im Oktober 2008 haben sich in einer weiteren Forsa-Umfrage weit über die Hälfte der Befragten für eine (Teil-)Verstaatlichung der Energiewirtschaft, der Banken und Versicherungen sowie des Flug-, Bahn- und Postverkehrs ausgesprochen (Tabelle 1). 
Darüber hinaus zeugen auch die Aktivitäten der Regierungen - in Deutschland und andernorts - von einer gesteigerten Bereitschaft, in die Funktionsweise der kapitalistischen Ökonomie wieder stärker zu intervenieren.

(1) So wurden in den letzten Monaten umfangreiche - international zum Teil abgestimmte - Rettungspakete verabschiedet, um das Kreditsystem zu stabilisieren. Sie enthalten unterschiedliche Elemente wie eine direkte Staatsbeteiligung, Bürgschaften und spezifische Auflagen für das Bankenmanagement.

(2) In Ergänzung hierzu ist ein internationaler Diskussions- und Verhandlungsprozess eingeleitet worden, der - von einigen sehr emphatisch als Ruf nach einem Bretton-Woods-System II eingefordert - eine gewisse Re-Regulierung der Finanzmärkte zum Gegenstand hat.

(3) Um der Gefahr einer sehr tief greifenden Wirtschaftskrise vorzubeugen, sind in fast allen Ländern Konjunkturprogramme aufgelegt worden, deren Erfolgsaussichten allerdings vorerst ungewiss bleiben.

In all diesen Aktivitäten spiegelt sich nicht zuletzt die negative Erinnerung an die Fragmentierung der Weltwirtschaft und die tiefe Wirtschaftskrise der 1930er Jahre. Um ein ähnliches Szenario heute zu vermeiden, ist es daher wenig verwunderlich, wenn Wissenschaftler, Journalisten und Politiker erneut keynesianische Überlegungen und Konzeptionen in die Debatte einbringen.

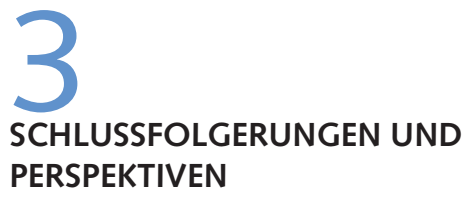

Die bisherigen Ausführungen lassen sich dahingehend zusammenfassen, dass der Entstaatlichungsprozess mehrere Phasen durchlaufen hat. Nachdem er im Rahmen des Leitbilds des schlanken Wettbewerbsstaates programmatisch offensiv proklamiert, in der Praxis jedoch nur schleppend vorangetrieben wurde, beschleunigte sich die Entstaatlichung im Kontext der neuen Paradigmen des aktivierenden Staates und Gewährleistungsstaates. Für die konkrete Organisation der Entstaatlichungspolitik war dieser Wandel des staatlichen Leitbildes keineswegs bedeutungslos. Noch wichtiger war jedoch, dass sich im Laufe der 1980er und 1990er Jahre die politischinstitutionellen und politökonomischen Bedingungen der gesellschaftlichen Reproduktion auch im globalen und europäischen Maßstab verändert hatten. Was die politisch-institutionellen Bedingungen betrifft, so vollzog sich ein umfassender staatlicher Transformationsprozess, der insbesondere durch drei Trends bestimmt war:

- durch die Denationalisierung, d. h. die territoriale Entgrenzung staatlicher Strukturen;

- durch die Verlagerung von Government zu Governance, d. h. eine netzwerkvermittelte Enthierarchisierung der Politik;

- durch die Internationalisierung von policy-regimes, also eine stärkere Einbindung der ehemals national definierten Politik in internationale bzw. globale Regulationskomplexe (Jessop 2002, S. 193ff.; Hirsch 2000).

Das Leitbild des Gewährleistungsstaates entsprach nicht nur den europäisch veränderten Kooperationsformen und Regulierungsvorgaben. Es schien auch in besonderem Maße den neuen Anforderungen der globalisierten politökonomischen Reproduktionsmuster zu genügen. Nicht ohne Grund wurde seit einiger Zeit davon gesprochen, dass sich mit dem Übergang zu einem ,finanzgetriebenen Akkumulationsregime“ (Boyer 2000) oder „Finanzmarktkapitalismus" (Windolf 2005) im Wirtschaftsprozess selbst die Machtbeziehungen und Investitionskriterien verschoben hatten. Schließlich hatten die Finanzmarktakteure - insbesondere die institutionellen Anleger (Versicherungen oder Investment- und Pensionsfonds), RatingAgenturen und Investmentbanken - vermittelt über die Wertpapiermärkte ihren Einfluss auf andere Bereiche der Ökonomie ausgedehnt. Zugleich sind im Zuge der Privatisierung und Kommerzialisierung öffentlicher Leistungen aber auch die Verfahren der demokratischen Kontrolle und Partizipation geschwächt und zumindest partiell - durch parallele Machtnetzwerke unter Einschluss von Politikberatern, Topmanagern, Consulting-Agenturen und Finanzmarktakteuren (Shareholdern) ersetzt worden (Crouch 2008, S. 119ff.).
Ob und wie sich die politischen Organisationsnetzwerke vor dem Hintergrund der öffentlichen Privatisierungskritik sowie der Finanzmarkt- und Weltwirtschaftskrise erneut verändern, ist derzeit noch unklar. Einiges spricht dafür, dass bereits jetzt zentrale Aspekte des finanzgetriebenen Akkumulationsregimes bzw. des angloamerikanischen Kapitalismusmodells infrage gestellt werden. Zum einen ist das Investmentbanking, das vormalige Aushängeschild des US-amerikanischen Finanzsystems, in eine tiefe Krise geraten. Die großen fünf Investmentbanken sind vorläufig von der Bildfläche verschwunden; ${ }^{1}$ und auch in Europa sind die Universalbanken geneigt, das Investmentbanking auf ein Minimum zu reduzieren. Zum anderen ist die erst kürzlich erfolgte Umstellung der Rechnungslegungsstandards von historischen Anschaffungswerten zur aktuellen Marktbewertung (fair value accounting) partiell wieder rückgängig gemacht worden, um der Unterbewertung der Unternehmensvermögen und damit der Gefahr einer Überschuldung entgegenzuwirken (Nölke 2009).

Diese Beispiele wie auch die verschiedenen Instrumente der ad hoc Krisenintervention verdeutlichen, dass in der Krise des globalen Finanzmarktkapitalismus einiges in Bewegung geraten ist. So verändern sich nicht nur die gesellschaftlichen Machtverhältnisse, auch die staatlichen Regulationsund Interventionsformen werden neu definiert. Allerdings wäre es naiv, bereits die Bereitschaft für ein umfassendes staatliches Interventionsmanagement durchweg positiv zu bewerten. Schließlich ist es, wenn man den Staat mit Nicos Poulantzas (1978, S. 119ff.) als materielle Verdichtung sozialer Kräfteverhältnisse begreift - einschließlich der spezifischen institutionellen Strukturen und Selektivitäten -, immer auch notwendig, nach dem sozialen Charakter und Zweck des Staatshandelns zu fragen.

Die öffentliche Debatte ist dabei vor allem durch zwei konkurrierende Leitbilder

\footnotetext{
Nach der Rettung von Bear Stearns durch JP Morgan Chase, der Insolvenz von Lehman Brothers und der Übernahme von Merrill Lynch durch die Bank of America haben im September 2008 sogar Goldman Sachs und Morgan Stanley beschlossen, sich in eine normale Geschäftsbank zu verwandeln und damit der Bankenaufsicht der Fed zu unterwerfen, um staatliche Zusatzkredite in Anspruch nehmen zu können.
} 
geprägt. Auf der einen Seite stehen die Befürworter des schlanken Wettbewerbsstaates wie beispielsweise Josef Ackermann oder Roland Koch (2008), die angesichts der prognostizierten Krisenperspektive den Staat auch als Katastrophenschützer, nicht aber als Wirtschaftsgestalter, gefragt sehen. Der Staat soll sich entsprechend darauf konzentrieren, die Ökonomie zu stabilisieren, um sich jedoch baldmöglichst wieder aus ihr zurückzuziehen und übermäßigen sozialen Ansprüchen und einer Politisierung des Wirtschafts- und Finanzsystems entgegenzutreten. Auf der anderen Seite wird von Gewerkschaften und sozial orientierten Politikern genau diese Vorstellung, nach der in der aktuellen Krise die Unternehmensverluste sozialisiert werden, um schon bald wieder die Demontage des öffentlichen Sektors voranzutreiben, scharf kritisiert. Eingefordert wird stattdessen ein demokratischer Sozial- und Investitions- staat, der allein schon durch die Schließung der bestehenden Investitionslücke - von etwa 50 bis $100 \mathrm{Mrd}$ € gemessen am EUDurchschnitt der öffentlichen Investitionsund Bildungsausgaben (vgl. den Beitrag von Truger in diesem Heft) - konjunkturpolitisch gegensteuern und die Perspektive einer demokratischen, sozialintegrativen und ökologisch nachhaltigen Reformpolitik eröffnen könnte (Bsirske 2008).

\section{LITERATUR}

Bandemer, St. von/Blanke, B./Hilbert, J./Schmid, J. (1995): Staatsaufgaben - von der schleichenden Privatisierung zum "aktivierenden Staat“, in: Behrens, F. et al. (Hrsg.): Den Staat neu denken. Reformperspektiven für die Landesverwaltungen, Berlin, S. 41-60

Bell, D. (1991): Die kulturellen Widersprüche im Kapitalismus, Frankfurt a. M./New York

Bieling, H.-J. (2008): Liberalisierung und Privatisierung in Deutschland: Versuch einer Zwischenbilanz, in: WSI-Mitteilungen 10, S. 541-547 Bieling, H.-J./Deckwirth, Ch./Schmalz, St. (Hrsg.) (2008): Liberalisierung und Privatisierung in Europa. Die Reorganisation der öffentlichen Infrastruktur in der Europäischen Union, Münster

Blanke, B./Bandemer, St. von (1999): Der "aktivierende Staat“ "in: Gewerkschaftliche Monatshefte 6, S. 321-330

Bofinger, P. (2008): Das Jahrzehnt der Entstaatlichung, in: WSI-Mitteilungen 7, S. 351-357

Boyer, R. (2000): Is a finance-led growth regime a viable alternative to Fordism? A preliminary analysis, in: Economy and Society 1, S. 111-145 Brandt, T./Schulten, Th./Sterkel, G./Wiedemuth, J. (Hrsg.) (2008): Europa im Ausverkauf. Liberalisierung und Privatisierung öffentlicher Dienstleistungen und ihre Folgen für die Tarifpolitik, Hamburg

Bsirske, F. (2008): Die Richtung ändern, in: ver.di Publik, November

Cockett, R. (1994): Thinking the Unthinkable. Think-Tanks and the Economic Counter-Revolution, 1931-1983, London

Crouch, C. (2008): Postdemokratie, Frankfurt a. M.

Dräger, K. (2004): Radikale Sozialstaatsreform in Europa, in: Das Argument 3-4, S. 505-515

Engartner, T. (2008): Privatisierung und Liberalisierung - Strategien zur Selbstentmachtung des öffentlichen Sektors, in: Butterwegge, Ch./Lösch, B./Ptak, R. (Hrsg.): Kritik des Neoliberalismus, Wiesbaden, S. 87-133 Europäische Kommission (2008): Statistischer Anhang zu Europäische Wirtschaft Herbst 2008, Luxemburg
Giddens, A. (1994): Beyond Left and Right. The Future of Radical Politics, Cambridge, Oxford

Gramsci, A. (1999-2001): Gefängnishefte, 10 Bd., Hamburg

Habermas, J. (1973): Legitimationsprobleme im Spätkapitalismus, Frankfurt a. M.

Habermas, J. (2008): Nach dem Bankrott. Der Privatisierungswahn ist an sein Ende gekommen. Nicht der Markt, sondern die Politik ist für das Gemeinwohl zuständig, Interview in "Die Zeit“ vom 6. November, S. 53-54 Hall, St. (1989): Antonio Gramscis Erneuerung des Marxismus und ihre Bedeutung für die Erforschung von „Rasse" und Ethnizität, in: Ders.: Ausgewählte Schriften. Ideologie, Kultur, Medien, Neue Rechte, Rassismus, Hamburg/Berlin, S. 56-91

Hennis, W. (1976): Legitimität. Zu einer Kategorie der bürgerlichen Gesellschaft, in: Kielmansegg, P. G. (Hrsg.): Legitimationsprobleme politischer Systeme, PVS-Sonderheft 7, Opladen, S. 9-38

Hirsch, J. (1998): Vom Sicherheitsstaat zum nationalen Wettbewerbsstaat, Berlin/Amsterdam

Hirsch, J. (2000): Die Internationalisierung des Staates. Anmerkungen zu einigen aktuellen Fragen der Staatstheorie, in: Das Argument 3, S. 325339

Jessop, B. (1993): Towards a Schumpeterian workfare state? Preliminary remarks on post-Fordist political economy, in: Studies in Political Economy 1, S. 7-39

Jessop, B. (2002): The Future of the Capitalist State, Cambridge Kannankulam, J. (2008): Autoritärer Etatismus im Neoliberalismus. Zur Staatstheorie von Nicos Poulantzas, Hamburg

Koch, R. (2008): Versagt hat nicht die Marktwirtschaft, in: Frankfurter Allgemeine Zeitung vom 22. Oktober

Kosellek, R. (1973): Kritik und Krise, Frankfurt a. M.

Lutz, B. (1984): Der kurze Traum immerwährender Prosperität, Frankfurt a. M./New York 
Majone, G. (1996): The Rise of the Regulatory State in Europe, in: West European Politics 3, S. 77-101

Matz, U. (1978): Zur Legitimität der westlichen Demokratie, in: Kielmansegg, P. G./Matz, U. (Hrsg.): Die Rechtfertigung politischer Herrschaft: Doktrinen und Verfahren in Ost und West, Freiburg; München, S. 27-58 Mittendorf, V. (2008): Bürgerbegehren und Volksentscheide gegen Privatisierungen und die Rolle der Gewerkschaften, in: Brandt, T./Schulten, T./Wiedemuth, J. (Hrsg.): Europa im Ausverkauf. Liberalisierung und Privatisierung öffentlicher Dienstleistungen und ihre Folgen für die Tarifpolitik, Hamburg, S. 310-329

Nölke, A. (2009): Finanzkrise, Finanzialisierung und die kapitalistische Vielfalt, in: Zeitschrift für Internationale Beziehungen 1, i. E.

Offe, C. (1984): Strukturprobleme des kapitalistischen Staates. Aufsätze zur politischen Soziologie, Frankfurt a. M.

Plehwe, D./Walpen, B. (1999): Wissenschaftliche und wissenschaftspolitische Produktionsweisen im Neoliberalismus. Beiträge der Mont Pélerin Society und marktradikaler Think Tanks zur Hegemoniegewinnung und -erhaltung, in: Prokla 2, S. 203-235
Polanyi, K. (1977) [1944]: The Great Transformation. Politische und ökonomische Ursprünge von Gesellschaften und Wirtschaftssystemen, Wien Poulantzas, N. (1978): Staatstheorie. Politischer Überbau, Ideologie, Sozialistische Demokratie, Hamburg

Ruggie, J. G. (1982): International Regimes, Transactions and Change: Embedded Liberalism in the Postwar Economic Order, in: International Organization 2, S. 379-415

Schneider, H. (1992): Europäische Integration: die Leitbilder und die Politik, in: Kreile, M. (Hrsg.): Die Integration Europas, PVS-Sonderheft 23, Opladen, S. 3-35

Schuppert, G. F. (2008): Der Gewährleistungsstaat. Zum Wandel der Staatlichkeit im Spiegel sich wandelnder Staatsbilder, in: Vorgänge 2, S. $14-25$

Steil, A. (1993): Krisen-Semantik. Wissenssoziologische Studien zu einem Topos moderner Zeiterfahrung, Opladen

Windolf, P. (Hrsg.) (2005): Finanzmarkt-Kapitalismus. Analysen zum Wandel von Produktionsregimen, Kölner Zeitschrift für Soziologie und Sozialpsychologie (KZfSS), Sonderheft 45, Wiesbaden 